\title{
Non-obvious influences on perception-action abilities
}

\author{
Michael T. Turvey ${ }^{1,2} \cdot$ Adam Sheya $^{3}$
}

Published online: 10 February 2017

(C) Psychonomic Society, Inc. 2017

\begin{abstract}
The sciences of development and learning have been slow to acknowledge that absence of an identifiable experience that relates straightforwardly to a given perceptionaction ability need not mean that experience per se is irrelevant to the emergence of that ability. A recent study reveals that a difference in diet (plain vs. energy rich) leads to a difference in how rats navigate (use of geometry vs. use of features, respectively). It is a good example of how a seemingly unrelated experience (e.g., what the rats eat) can be a non-obvious yet crucial determiner of perception-action modes. We situate this finding in the broader context of the related conceptions of Schneirla's and Lehrman's Developmental Systems Theory, Gottlieb's Probabilistic Epigenesis, and Bolles's Structure of Learning (see article for references). In doing so we highlight that such phenomena may be the norm, both in development and learning, rather than the exception.
\end{abstract}

Keywords Development $\cdot$ Learning $\cdot$ Perception-action

\section{Introduction}

A traditional bias is to assume that a candidate experiential precursor to a spatial perceptual ability is an experience that can be linked to the ability by a plausible, rational argument.

Adam Sheya

adam.sheya@uconn.edu

1 Center for the Ecological Study of Perception and Action, University of Connecticut, Storrs, CT, USA

2 Haskins Laboratories, New Haven, CT, USA

3 Department of Psychological Sciences, University of Connecticut, Storrs, CT, USA
An experience that bears no obvious, transparent, easily rationalized, relation to the spatial perceptual ability is presumed to be an unlikely precursor to the perceptual ability on prima facie grounds. A recent example of the latter is a report by Tran and Westbrook (2015) that the use of spatial geometry (distance and direction) by rats depends on their diet. Rats fed regular chow related to their surroundings by means of geometry. Rats fed an energy-rich diet did not; they related to their surroundings by means of features (luminance and pattern). We wish to underscore the broad theoretical significance of such findings. We do so primarily in the context of ideas that ground the perspective on development referred to as Developmental Systems Theory (e.g., Lehrman 1970; Schneirla, 1961, 1966; Turkewitz, 1994) or Probabilistic Epigenesis (e.g., Anderson, Hubbard, Campos, et al. 2000; Gottlieb, 2007; Johnston, 1997; Lickliter \& Honeycutt, 2003; Miller, 1988) allied with Bolles's (1975/1979) Structure of Learning.

\section{Absent rational precursors}

In the domain of developmental sciences, where rational precursors of a given ability have not been identified or are deemed impossible, the historical tendency has been to regard the ability as genetically determined (that is, unlearned, innate). The developmental sciences have slowly come to appreciate, however, that the lack of identifiable experiences that relate to a given perception-action ability in a straightforward manner does not mean that experience is irrelevant to the emergence of that ability. Unlearned or so-called "instinctive" abilities - those commonly characterized as not traceable to straightforward learning or practice - can be experientially based, but in a non-obvious way (Gottlieb, 1992, 1997; see also Anderson et al. 2000). 
Consider the following nonobvious influences on highly stereotypic perception-action abilities. (a) Squirrel monkeys fed during rearing with live insects develop the speciesspecific avoidance behavior toward snakes. If they are instead fed fruit or chow, the fear of snakes fails to emerge (Masataka, 1994). (b) Male brown-headed cowbirds sing. The females do not. The females, however, are "in charge" of insuring the geographical variation in cowbird song. They implement their duty by optical means, through timely wing strokes visible to the male that are simultaneously sexually significant and instructional. These visual displays constitute a non-obvious means to bring the maturing male's singing in compliance with the regional dialect (King \& West, 1983; Smith, King, \& West, 2000; West \& King, 1988). In brief, no pedagogical female visual displays, no region-specific brown-head cowbird song. (c) Honey bee larvae incubated at a lower temperature than normal do not differ as adults in within-hive behavior but do differ in the post-foraging return-to-hive dance that communicates a food source. Compared to bees incubated at the normal temperature, their waggle dance is significantly reduced in the likelihood of occurring, number of circuits, and duration of the waggle phase (Tautz, Maier, Groh, Rossler, \& Brockman, 2003).

To facilitate appreciation of non-obvious influences we consider two examples in detail, specifically, two experiments by Wallman (1979) conducted with White Leghorn chicks. There were two groups of chicks in each of two experiments. In the first experiment, the chicks in one group wore white mittens; they could not see their toes. For simplicity, the two groups can be referred to as "occluded toes" and "visible toes," respectively. Individuals in the two groups were raised in individual chambers on Days 1 and 2 with water but no food (no edibles to spill on the floor, nothing to peck at). Testing was on Days 3 and 4, conducted in new but identical-in-design chambers. Individual chicks in both groups were individually tested with a single mealworm. Pecks at the mealworm were either exploratory or ingestive. "Occluded toes" visually fixated on the mealworm more quickly than and three times longer than "visible toes". "Occluded toes" approached more slowly than, and pecked from a greater distance than "visible toes" (but even with maximum extension of neck, "occluded toes" barely reached the mealworm). Whereas "visible toes" engaged in mainly ingestive pecks, "nonvisible toes" engaged in mainly exploratory pecks. More significantly, whereas "visible toes" ate the mealworm, "nonvisible toes" did not.

Wallman's second experiment with the same design was conducted with toes visible in their natural color for one group and likewise visible, but painted black, for the other group. The mealworm was fixated longer, pecked at more often without being picked up, and picked up less frequently by the chicks with black-painted toes than by the chicks with naturally colored toes.
The implication of the foregoing examples is that there is an experiential context, a possibly dense network of experiences, which entails the emergence of stereotypic snake avoidance, stereotypic cowbird singing, stereotypic bee communication, and stereotypic mealworm eating. The stereotypic behaviors fail to emerge when aspects of the context, however subtle, however disingenuous, are omitted. Such is similarly the case for the stereotypic development of the somatosensory nervous system: it does not occur when aspects of the context, as subtle and disingenuous as twitching when sleeping, are omitted (e.g., Khazipov, Sirota, Leinekugel, Holmes, et al., 2004; Marques, Imtiaz, Iida, \& Pfeifer, 2013; Petersson, Waldenstrom, Fahraeus, \& Schouenborg, 2003). Arguably, such phenomena are tied to a complex system's characteristic properties: a non-trivial spread of causes, an absence of a predefined endpoint, a nesting of timescales, and a coupling of heterogeneous components (Sheya \& Smith, 2010).

\section{Dissecting causal structure}

Killeen and Nash's (2003) dissection of causal structure helps one's appreciation of the phenomena in question. A causal context $C$ will bring about an effect $E$ if $C$ contains all the necessary causes and at least one sufficient cause. An efficient cause labeled sufficient is one that precedes and initiates a state change. An efficient cause labeled necessary is one whose absence from an otherwise sufficient $C$ disallows the expected state change. Generally, a $C$ sufficient for producing $E$ provides many necessary causes that are so indirectly related to $E$ that they go unnoticed and unnamed. Moreover, they are- - by virtue of their normalcy, their commonness-taken for granted. In the above examples, sufficient causes for triggering stereotypic snake avoidance, stereotypic cowbird singing, stereotypic bee waggle dance, and stereotypic mealworm eating were present. What were absent, apparently, were the necessary causes of previously eating live insects, timely female wing motions, previously incubating at a specific temperature, and previously seeing one's toes, respectively.

\section{Span of non-obvious experiences}

The non-obvious experiences supporting the emergence of a species-specific behavior span the prenatal, perinatal, and postnatal periods. The significance of perinatal experiences has been revealed in studies on the responses of mallard ducklings to the maternal assembly and alarm calls (Gottlieb, 1997; Miller, 1997). Mallard ducklings begin to vocalize 2-3 days before hatching. If the self-produced sounds are eliminated by temporarily closing off the embryo's vocal chords, then the probability that the hatched duckling will respond selectively to the mother's postnatal call to assemble (for departure from 
the nest) will be significantly reduced. For a duckling with operative vocal chords, the absence of perinatal sounds arising from within the nearby eggs of sibling embryos can also reduce the probability of subsequent selective responding to the mother's assembly call. Importantly for matters of interpretation, the specifics of the self- and sibling-vocalizations are crucial to the effects (Gottlieb, 1997). It is these missing specifics of perinatal hearing, not simply the absence of opportunities for auditory stimulation, that result in the reduced likelihood of selectively responding after birth to the maternal assembly call of one's species and not to that of another species. ${ }^{1}$

A similar conclusion has been reached for the freezing behavior that occurs in ducklings as a response to the maternal alarm call - a response that, like the response to the assembly call, occurs when the call is heard for the very first time. The investigation of the perinatal and postnatal influences on the freezing response reveals, additionally and importantly, what can only be termed "unreasonable" relations between the nonobvious auditory and social experiences (Miller, 1988, 1997). As argued by Gottlieb (e.g., 1997, 1998, 2000), addressing these "unreasonable" matters requires a probabilistic epigenesis perspective (see Fig. 1) that respects (a) the reciprocity of influences within and between levels of an organism's developmental manifold (constituted as genetic, neural, behavioral, and environmental dynamics), and (b) the ubiquity of geneenvironment interaction dynamics in the realization of all phenotypes.

Figure 1 fosters appreciation for the interactive nature of the dynamic relations among the levels that collectively beget the development of neural and somatic structure and function, perceptual abilities, and behavior. It also fosters appreciation for the core of the non-obvious nature of development, viz., the generic nonlinear interconnectedness of parts and processes that challenge the intellectual endeavor of deriving developmental causes from effects. ${ }^{2}$ Valsiner (2007) recommends that the upward and downward arrows indexing causal linkages in Fig. 1 be viewed as "tubes" that link local field-like behavior at any one level at any given time to local field-like behavior at one or more of the other levels. Focusing on the behavioral trajectory as an example, Fig. 1 implies that at any intersection, direct or mediated, of the genetic, neural, and

\footnotetext{
$\overline{1}$ Gottlieb (1997, p. 270) reports a parallel observation with mallard ducks. Eggs were incubated at a lower temperature than normal as part of an investigation into the significance of the embryonic heart beat rate to the rate of the maternal call preferred by the ducks when hatched. No change in the preferred rate of the maternal call was observed but there was a change in respect to the component frequencies. The newly hatched preferred a maternal call with the higher frequencies deleted.

${ }^{2}$ Inference from effect to cause is abductive inference. The conventional means of implementing abduction is Bayesian. It is associated with a recalcitrant problem. In Glymour's (2001, p. 97) words: “[n]o correct, feasible, general Bayesian procedure is known that provides correct information-in the large sample limit-about the existence of unobserved common causes of observed variables."
}

environmental levels with the behavioral trajectory, there is a consequent behavioral dynamic that is conditional on the specifics of the four trajectories and their interactions with each other.

The developmental system as depicted may be considered a frustrated system. In the physics of soft-matter, a frustrated system is one that has no well-defined ground state simply because competing interactions among the system's components cannot simultaneously be at an energy minimum. Arguably, most non-trivial behaviors in a many body system (a system with multiple degrees of freedom) originate in some sort of frustration, whether the system is physical or biological. The frustration is tied to the fact that in the course of self-organizing - that is, in the process of minimizing and/or adapting to the local non-equilibrium dynamics-new degrees of freedom emerge to be coordinated.

\section{Issue of releasers}

The "releaser" or "sign stimulus" foundational to ethology would seem to be a prime example of a non-obvious and unreasonable phenomenon. Unfortunately, there are reasons to think otherwise. A prominent exemplar of releaser is in respect to "food begging" by the herring gull chick. Tinbergen and Perdeck's (1950) presentation and discussion of the stimulus features that elicit (i.e., release) begging for food involve the construction of various three-dimensional models of the parent's head (white) and bill (yellow with a red spot) together with an additional artificial stimulus consisting of a red knitting needle with three white bands (see Fig. 2). The non-intuitive unreasonable finding was that the red knitting needle with three white bands elicited a stronger response (126 pecks vs. 100 pecks) than the more accurate (realistic) three-dimensional models (Tinbergen \& Perdeck, 1950). It was as if the red needle captured the essential nature of the environmental condition which releases the "food begging" behavior. Tinbergen (1951) came to refer to such environmental conditions as supernormal releasers or supernormal sign-stimuli on the basis that they elicit (paradoxically) a larger response than the putatively more realistic models. ${ }^{3}$ A careful and respectful critical examination by ten Caté (2009) (see also ten Caté, Bruins, den Ouden, et al. 2009) of Tinbergen's various reports has found the experimental

\footnotetext{
${ }^{3}$ A human parallel to this eating-related phenomenon has been suggested, such as the consumption of so-called psychoactive substances (e.g., high caloric foods and alcohol). To the latter supernormal condition can be added media inculcated in entertainment, pornography, gaming, and retail products. It has been suggested that all such supernormal conditions amplify the activity of the underlying neuro-chemical systems beyond their original evolutionary charge (cf. Goodwin et al., 2016; Meshi et al, 2015; Ward, 2013).
} 


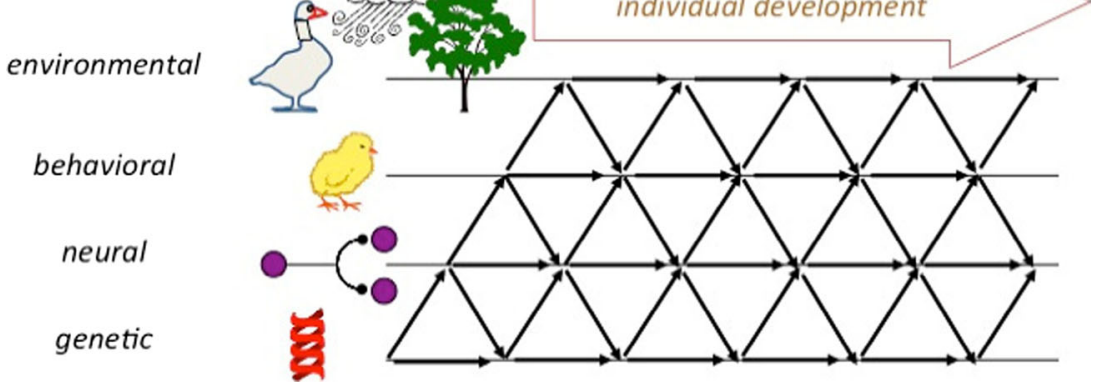

Fig. 1 Gottlieb's probabilistic epigenetic framework: Each level influences, and is influenced by, every other level with greater or lesser probability (see text for details). Figure 1 is modeled after Fig. 14-3 in
Gottlieb (1992); it differs in the addition of the images representing the four levels and the arrow that depicts the direction of developmental time foundation for Tinbergen's identification of releasers, normal and supernormal, to be open to question. The vagaries of the experimental observations are conveyed by (a) the different conclusions Tinbergen drew from his assembling of the data in different ways from one publication to the next, and (b) the belatedly realized fact that contrasting candidates for releasers were not presented an equal number of times. In respect to Fig. 2, the feature "red" in either model was found to be irrelevant in later presentations of the data. As ten Caté (2009) discerned, in Tinbergen's publications exploratory experiments assumed the status of experimental findings. Ten Caté's conclusion is that the majority of Tinbergen's publications on the herring gull were presented primarily as an

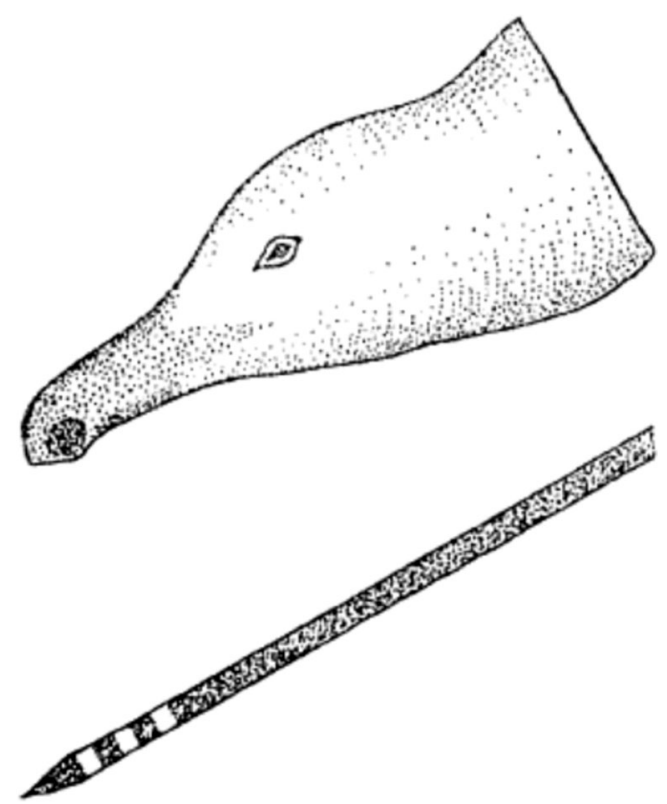

Fig. 2 Top: a gypsum model in the natural shape of a Herring Gull's head as a normal releaser. Bottom: A red stick with white rings as a supernormal releaser (Figure 33 in Tinbergen \& Perdeck, 1950) illustration of how one might conceptualize matters in the ethological perspective.

\section{Non-obvious variables in learning and memory}

Returning to Tran and Westbrook (2015), it can be said that they did find "unreasonable" phenomena. Enriched chow disposed the experimental animals to relating to their environments in terms of luminance and pattern rather than in terms of the more dominant distance and direction. Though "unreasonable" in the broader viewpoint, Tran and Westbrook's investigation was inspired by studies suggesting that the architecture of the hippocampus instantiates both the luminance-and-pattern mode of orienting and distance-anddirection mode of orienting, and that the hippocampus, architecturally and functionally, is influenced by diet (e.g., Kanoski, Meisel, Mullins, \& Davidson, 2007). Their "unreasonable" relation is reasonably motivated by empirical facts, which is not to say, however, that it is reasonably motivated conceptually and theoretically. Absent is the logic and theory that would account for the fact that a diet of high fat and high sugar should selectively impair neural machinery involved in the perception of distance and direction.

Tran and Westbrook's finding was within a study that, in contrast to the cases discussed above, would be defined conventionally as focused on learning more so than on development. The experimental animals were familiarized with the apparatus over a period of 21 days during which they were fed either one of the two diets. They were then tested within that familiar apparatus but with the special experimental conditions defining either a placerecognition test or an object-recognition test. Although it is rarely (if ever) noted in the discourse on non-obvious variables in development, the experimental inquiry into learning and memory is likewise confronted by variables that resist rationalization. 
For the memory experimentalist (e.g., Capaldi \& Neath, 1995; Wickens, 1987), the setup of an experiment divides into (a) variables distributed over organism and environment that seemingly bear no causal relation to the experimental task whatsoever (so called, context $\alpha$ ) and (b) variables that are seemingly obvious in their relation to the experimental task (so called context $\beta$ ). Typically, only the rationally transparent variables (context $\beta$ ) are identified as context. An experiment by Overton (1964) exemplifies an internal manipulation of context $\alpha$ (see Capaldi \& Neath, 1995). Rats infused with sodium pentobarbitol or saline solution learned to discriminate the arms of a T-maze. They performed near perfectly when later tested in the same drug state as the learning phase (e.g., sodium pentobarbital-sodium pentobarbital) but at chance when tested in the opposite drug state (sodium pentobarbital-saline).

Thomas (1985) highlights the explanatory problem of limiting one's perspective to context $\beta$. Pigeons that learned a color-discrimination problem when standing on a flat (inclined) surface failed the problem when the surface was inclined (flat). In this investigation, most of the changes from "study" to "test" were in the class of rationally opaque. The changes were primarily of angle of view and the angular relations among hips, knees, ankles, and digits. How should one proceed to explain what these changes have to do with the acquired color discrimination?

As intimated above, the explanatory significance of nonobvious rationally opaque variables to the operative context is a defining characteristic of the probabilistic epigenetic approach to development (Gottlieb, 1997; Johnston, 1997; Miller, 1997). For example, the freezing response exhibited by ducklings to the maternal alarm call is linked developmentally, and non-obviously, to their own perinatal (in the shell, prior to hatching) vocalizations. Absence of the latter (experimentally induced by temporary devocalization) can eliminate the adaptive freezing behavior normally seen in hatched ducklings (for a summary see Miller, 1997). From a focus on identifying precursors rationally related to outcomes, the finding by Miller is prima facie implausible: "There is no coherent rational argument that might lead one to suppose that the duckling has to hear its own embryonic call before showing an adaptive response to the alarm call (Johnston, 1997, p. 511)."

In important respects, the decline in what had been a commonplace acceptance during most of the 20th century of the laws of learning as uniform and universal (the principle of equipotentiality) was motivated by findings that learning depended non-trivially upon a triad of kinds: organism kind, behavior kind, and situation kind (Bolles, 1975/1979; Bouton \& Fanselow, 1997). The empirical counters to uniformity were attributed to "constraints on learning," but as Bolles (1975/1979) underscored, the "constraints" are only half the story. There are also "facilitations of learning." Taken together, the observed constraints and facilitations suggest (a) that behavior is adaptive, functional, mostly unlearned, and typically a solution to a problem, and (b) that learning is an adaptation that is mostly in terms of perceiving and mostly of affordances. The latter concept central to J. J. Gibson's (1979/1986) ecological formulation of perception and action can be defined as follows: A given situation $X$ affords a given activity $Y$ for a given organism $Z$ on a given occasion $O$ if and only if the situation $X$ and the organism $Z$ are mutually compatible on dimensions of relevance to the activity $Y$ (e.g., Turvey \& Shaw, 1979; Turvey, 2015).

The significance of affordance to behavior and learning is brought home by what Bolles (1975) regarded as one of the most troublesome problems faced by learning theory, past and present: whereas some avoidance responses are acquired very rapidly, others are acquired very slowly, if at all. A prime example is that of rats learning to avoid shock by running but not learning to avoid shock by standing on their hind legs (Bolles, 1975, pp. 194-196). The foregoing was the case even though running and standing had the same high incidence of occurrence on the initial trials of the experiment. The situation $X$ and the animal $Z$ were mutually compatible on dimensions relevant to $Y$ as running but not $Y$ as standing. The situation afforded running for the animal and was perceived as such. Running was learned. The situation did not afford standing upright for the animal and was perceived as such. Standing upright was not learned, despite being a behavior that the animal readily enacts. With additional cases of like kind in mind, Bolles (1975, p. 196) was drawn to the conclusion that "The solution of an avoidance problem seems to depend on how the animal perceives the situation."

The noted affordances in the preceding example are reasonably obvious in the sense that they are relatively easy to put into words. This will rarely be the case. Consider what affords the lizard Anolis perching-for-predating. It is a place that is view-from-able, jump-from-able with accuracy, and sprintupon-able with maximal speed, with each of the foregoing features a function of the surrounding vegetation, the lizard's leg length, the branch's breadth, and the branch's texture (Irschick \& Losos, 1999; Moermond, 1979). All properties conjoin to define this particular affordance. In a formal account one would want to refer to it as a single variable, albeit a compound (e.g., Turvey, 2015, Section 3.3). As such, it would be a non-obvious variable.

\section{By way of conclusion}

We conclude with two well-motivated conclusions, albeit speculative. First, in respect to development and learning, necessary causes relate indirectly to outcome. Typically, these causes are commonplace, taken for granted, and unnoticed. The specific role that any such cause as experience or activity 
might play—such as inducting, facilitating, maintaining, or canalizing a behavior (Gottlieb, 1997) — is rarely, if ever, identified. Second, in respect to development and learning, all experiences in their respective time scales might be expected to contribute - the logically major and minor, the obvious and the non-obvious, the prolonged and the instantaneous, the recurring and the once only.

In these latter respects, the dynamics of development and learning may be very general. They may, for example, be akin to the dynamics of earthquakes. The latter follow power law behavior ${ }^{4}$ with negative slope (Gutenberg-Richter Law). Small earthquakes are frequent, medium-sized earthquakes are less so, and large earthquakes are rare. ${ }^{5}$ By the suggested analogy, in respect to developmental/learning experiences of relevance, the least obvious occur most often, the more obvious occur less often, and the most obvious occur least often.

\section{References}

Anderson, D. J., Hubbard, E. M., Campos, J. J., Barlu-Roth, M. A., Witherington, D., \& Hertenstein, M. J. (2000). Probabilistic epigenesis, experience, and psychological development in infancy. Infancy, $1,245-251$.

Bolles (1975/1979). Learning theory. New York, NY; Holt. Rinehart \& Winston.

Bouton, M. E., \& Fanselow, M. S. (1997). Learning, motivation and cognition. Washington, DC: American Psychological Association.

Capaldi, E. J., \& Neath, I. (1995). Remembering and forgetting as context discrimination. Learning \& Memory, 2, 107-132.

Gibson J. J. (1979/1986). The ecological approach to visual perception. Boston, MA: Houghton Mifflin.

Glymour, C. (2001). The mind's arrows: Bayes nets and graphical causal models in psychology. Cambridge: MIT Press.

Goodwin, B. C., Browne, M., Rockloff, M., \& Loxton, N. (2016). Rash impulsivity predicts lower anticipated pleasure response and a preference for the supernormal. Personality and Individual Differences, 94, 206-210.

Gottlieb, G. (1992). Individual development and evolution: The genesis of novel behavior. New York: Oxford University Press.

Gottlieb, G. (1997). Synthesizing nature and nurture: Prenatal roots of instinctive behavior. Mahwah: Lawrence Erlbaum Associates.

Gottlieb, G. (1998). Normally occurring environmental and behavioral influences on gene activity: From central dogma to probabilistic epigenesis. Psychological Review, 105, 792-802.

Gottlieb, G. (2000). Environmental and behavioral influences on gene activity. Current Directions in Psychological Science, 9(3), 93-97.

Gottlieb, G. (2007). Probabilistic epigenesis. Developmental Science, 10, $1-11$.

Helmstetter, A. (2003). Is earthquake triggering driven by small earthquakes? Physical Review Letters, 91, 058501.

Irschick, D. J., \& Losos, J. B. (1999). Do lizards avoid habitats in which their performance is submaximal? The relationship between

\footnotetext{
${ }^{4}$ Power-law behavior means (a) no characteristic measure (no defining mean) and (b) scale invariance (same dynamics at every scale).

${ }^{5}$ The more frequent earthquakes at smaller scales seemingly affect the distribution of elastic stresses that contribute to the manifestation of the less frequent earthquakes at larger scales (Helmstetter 2003; Marsan, 2005).
}

sprinting capabilities and structural habitat use in Caribbean Anoles. American Naturalist, 154, 293-305.

Johnston, T. D. (1997). Comment on Miller. In C. Dent-Read \& P. Zukow-Goldring (Eds.), Evolving explanations of development (pp. 509-513). Washington, DC: American Psychological Association.

Kanoski, S. E., Meisel, R. L., Mullins, A. J., \& Davidson, T. L. (2007). The effects of energy-rich diets on discrimination reversal learning and on BDNF in the hippocampus and prefrontal cortex of the rat. Behavioral Brain Research, 182(1), 57-66.

Khazipov, R., Sirota, A., Leinekugel, X., Holmes, G. L., Ben-Ari, Y., \& Buzsaki, G. (2004). Early motor activity drives spindle bursts in the developing somatosensory cortex. Nature, 432, 758-761.

Killeen, P., \& Nash, M. R. (2003). The four causes of hypnosis. International Journal of Clinical and Experimental Hypnosis, 51, 195-231.

King, A. P., \& West, M. J. (1983). Epigenesis of cowbird song: A joint endeavor of males and females. Nature, 305, 704-706.

Lehrman, D. S. (1970). Semantic and conceptual issues in the naturenurture problem. In L. R. Aronson, D. S. Lehrman, E. Tobach, \& X. S. Rosenblatt (Eds.), Development and evolution of behavior (pp. 17-52). San Francisco: Freeman.

Lickliter, R., \& Honeycutt, H. (2003). Developmental dynamics: Toward a biologically plausible evolutionary psychology. Psychological Bulletin, 129, 819-835.

Marques, H. G., Imtiaz, H. G., Iida, F., \& Pfeifer, R. (2013). Selforganization of reflexive behavior from spontaneous motor activity. Biological Cybernetics, 107, 25-37.

Marsan, D. (2005). The role of small earthquakes in redistributing crustal elastic stress. Geophysics Journal International, 163, 141-151.

Masataka, N. (1994). Effects of experience with live insects on the development of fear of snakes in squirrel monkeys, Saimiri sciureus. Animal Behavior, 46, 741-746.

Meshi, D., Tamir, D. I., \& Heekeren, H. R. (2015). The emerging neuroscience of social media. Trends in Cognitive Sciences, 19(12), 771782 .

Miller, D. (1988). Development of instinctive behavior: An epigenetic and ecological approach. In E. M. Blass (Ed.), Handbook of behavioral Handbook of behavioral neurobiology, Vol. 9: Developmental psychobiology and behavioral ecology (pp. 415-444). New York: Plenum.

Miller, D. (1997). The effects of nonobvious forms of experience on the development of instinctive behavior. In C. Dent-Read \& P. ZukowGoldring (Eds.), Evolving explanations of development (pp. 457507). Washington, DC: American Psychological Association.

Moermond, T. (1979). The influence of habitat structure on Anolis foraging behavior. Behaviour, 70, 147-167.

Overton, D. A. (1964). State-dependent of "dissociated" learning produced with pentobarbitol. Journal of Comparative and Physiological Psychology, 57, 3-12.

Petersson, P., Waldenstrom, A., Fahraeus, C., \& Schouenborg, J. (2003). Spontaneous muscle twitches during sleep guide spinal self-organization. Nature, 424, 72-75.

Schneirla, T. C. (1961). Instinctive behavior, maturation-Experience and development. In B. Kaplan \& S. Wapner (Eds.), Perspectives in psychological theory: Essays in honor of Heinz Werner (pp. 303334). New York: International Universities Press.

Sheya, A. \& Smith, L. (2010). Dynamics of the process of development. In C. Hooker (Ed.), Handbook of the Philosophy of Science. Volume 10: Philosophy of Complex Systems (pp. 605-627). Elsevier BV.

Smith, A. V., King, A. P., \& West, M. J. (2000). A role of her own: Female cowbirds, Molothrus ater, influence the development and outcome of song learning. Animal Behaviour, 60, 599-609.

Tautz, J., Maier, S., Groh, C., Rossler, W., \& Brockmann, A. (2003). Behavioral performance of honeybees is influenced by the 
temperature experienced during their pupal development. Proceedings of the National Academy of Sciences, USA, 100, $7343-7347$.

ten Cate, C. (2009). Niko Tinbergen and the red patch on the herring gull's beak. Animal Behaviour, 77(4), 785-794.

ten Cate, C., Bruins, W. S., den Ouden, J., Egberts, T., Neevel, H., Spierings, M., \& Brokerhof, A. W. (2009). Tinbergen revisited: A replication and extension of experiments on the beak colour preferences of herring gull chicks. Animal Behaviour, 77(4), 795-802.

Thomas, D. R. (1985). Contextual stimulus control of operant responding in pigeons. In P. Balsam \& A. Tomie (Eds.), Context and learning (pp. 295-322). Hillsdale: Erlbaum.

Tinbergen, N. (1951). The study of instinct. New York: Clarendon Press/ Oxford Press.

Tinbergen, N., \& Perdeck, A. C. (1950). On the stimulus situation releasing the begging response in the newly hatched herring gull chick (Larus argentatus Pont.). Behaviour, 3, 1-39.

Tran, M. D., \& Westbrook, R. F. (2015). Rats fed a diet rich in fats and sugars are impaired in the use of spatial geometry. Psychological Science, 26, 1947-1957.

Turkewitz, G. (1994). Sources of order for intersensory functioning. In D. J. Lewkowicz \& R. Lickliter (Eds.), The development of intersensory perception: Comparative perspectives (pp. 3-17). Hillsdale: Erlbaum.
Turvey, M. T. (2015). Quantum-like issues at nature's ecological scale (the scale of organisms and their environments). Mind \& Matter, 13, $7-44$.

Turvey, M. T., \& Shaw, R. (1979). The primacy of perceiving: An ecological reformulation of perception for understanding memory. In L.-G. Nilssen (Ed.), Perspectives on memory research: In honor of Uppsala University's 500th anniversary (pp. 167-222). Hillsdale: Erlbaum.

Valsiner, J. (2007). Gilbert Gottlieb's theory of probabilistic epigenesis: Probabilities and realities in development. Developmental Psychobiology, 49, 832-840.

Wallman, J. (1979). A minimal visual restriction experiment: Preventing chicks from seeing their feet affects later responses to mealworms. Developmental Psychobiology, 12, 391-397.

Ward, A. F. (2013). Supernormal: How the Internet is changing our memories and our minds. Psychological Inquiry, 24. 341-348.

West, M. J., \& King, A. P. (1988). Female visual displays affect the development of male song in the cowbirds. Nature, 334, 244 246.

Wickens, D. D. (1987). The dual meanings of context: Implications for research, theory, and applications. In D. S. Gorfein \& R. R. Hoffman (Eds.), Memory and learning: The Ebbinghaus Centennial Conference (pp. 135-152). Hillsdale: Erlbaum. 